\title{
Primary Catheter-Directed Thrombolysis For Porto-Mesenteric Venous Thrombosis (PMVT) In Non-Cirrhotic Patients.
}

\author{
Chia-Ling Chiang \\ Kaohsiung Veterans General Hospital \\ Huei-Lung Liang ( $\sim$ hlliang@vghks.gov.tw) \\ Kaohsiung Veterans General Hospital \\ Ming-Feng Li \\ Kaohsiung Veterans General Hospital
}

\section{Research Article}

Keywords: catheter directed thrombolysis (CDT), porto-mesenteric venous thrombosis (PMVT), percutaneous transhepatic route, non-cirrhotic patients, portal hypertension, ischemic bowel

Posted Date: November 1st, 2021

DOI: https://doi.org/10.21203/rs.3.rs-1003533/v1

License: () (1) This work is licensed under a Creative Commons Attribution 4.0 International License. Read Full License 


\section{Abstract}

Purpose: To report our technique, treatment strategy and clinical outcomes for porto-mesenteric venous thrombosis (PMVT) in non-cirrhotic patients.

Methods: 16 non-cirrhotic PMVT patients (mean age: 48.6 years) with imminent intestinal ischemia were enrolled from 2004-2020. 8 patients presented thrombus extension into the peripheral mesenteric vein, close to the venous arcade. Transhepatic catheterdirected thrombolysis (CDT) was performed by urokinase infusion (60,000-30,000 IU/hour concomitant with heparin 300-400 IU/hour), catheter aspiration and/or balloon dilation/stent placement. Additional intra-arterial mesenteric infusion of urokinase (30,000 IU/hour) was given in patients with the peripheral mesenteric venules involved. Transjugular intrahepatic porto-systemic shunt (TIPS) was created in patients with poor recanalization of the intrahepatic portal flow (PV).

Results: The transhepatic route was adopted in all patients, with adjunct indirect mesenteric arterial thrombolytic infusion in 8 patients. A total of up to 20.4 million IU urokinase was infused for 1-30 days' treatment duration. TIPS was created in 3 patients with recanalization failure of the intrahepatic PV. Technical success was achieved in $100 \%$ of patients with complete recanalization of $80 \%$ and partial recanalization of $20 \%$. No major procedure-related complications were encountered. The 30 -day mortality rate was $6.7 \%$. The overall 1 - and 2-year primary and secondary patency were both $74.0 \%$ and $84.6 \%$ respectively.

Conclusions: CDT can be performed as a primary salvage treatment once the diagnosis is made. CDT via the transhepatic route with tailored thrombolytic regimen is safe and effective for both acute and chronic PMVT. TIPS creation can be preserved in noncirrhotic PMVT patients if intrahepatic PV recanalization fails.

\section{Introduction}

Porto-mesenteric venous thrombosis (PMVT) is an uncommon cause of mesenteric ischemia, accounting for only $5-15 \%$ of cases $^{1}$. Its outcome is unpredictable, reflecting differences in clot distribution, clot volume, speed of onset and the presence of the radiological features of intestinal ischemia. Søgaard et al reported the 30-day mortality risk was particularly high for patients with mesenteric vein thrombosis $(63.1 \%)$ when compared with that of portal vein thrombosis $(15.6 \%)^{2}$. PMVT can be either acute, presenting commonly with abdominal pain, or chronic, presenting with features of portal hypertension. On rare occasions, it is diagnosed as an incidental finding on abdominal CT scans ${ }^{3}$. The aim of treatment is to reverse or prevent the advancement of diffuse thrombosis in the porto-mesenteric veins (PMV) and to treat its complications ${ }^{4}$. There are clear recommendations from the American Association for the Study Liver Diseases (AASLD) and European Association for the Study of the Liver (EASL) for the use of anticoagulation in non-cirrhotic acute PMVT ${ }^{5,6}$. However, anticoagulation alone in acute, extensive PMVT does not necessarily result in spontaneous clot lysis and leaves patients at risk of complications, including intestinal infarction and portal hypertension?

Several studies with small patient cohorts attempting the prompt restoration of the porto-mesenteric flow in the non-cirrhotic PMVT patients using either catheter-directed thrombolysis (CDT) alone or with the aid of thrombectomy devices have been reported in the literature ${ }^{7-15}$. These procedures were performed via the transhepatic, transjugular and/or indirect superior mesenteric artery routes. Nowadays, the transjugular route is advocated for this purpose because of the high percentage of major bleeding complications (50-60\%) of the transhepatic route ${ }^{9,15}$. However, high-dose thrombolytic regimens were both adopted in the above-mentioned studies. Also, entering the thrombosed portal vein via the transjugular route is technically more difficult, requiring more needle puncture passes, which may be accompanied by higher bleeding complications if intrahepatic arterial or capsular punctures occur. In addition, the rationale of creating a transjugular porto-systemic shunt (TIPS) in non-cirrhotic PMVT patients is still a matter of on-going debate. In a recent report, Benmassaoud et al proposed a stepwise thrombolysis regimen, applying local CDT in acute PMVT patients after the failure of initial systemic thrombolysis (ST), which occurred in $63 \%$ of their patients ${ }^{7}$. From the point of view of thrombus management, the earlier CDT is started, the higher the recanalization rate and the better the clinical outcomes can be achieved, as long as the technique of CDT is safe and easy to accomplish. Herein, we report our interventional technique, treatment strategy and clinical outcomes in managing non-cirrhotic PMVT with our pharmaco-lytic regimen by primary local CDT via the transhepatic route. 


\section{Patients and Methods}

Sixteen non-cirrhotic patients (male: 12, female: 4) with PMVT were enrolled from 2004 to 2020 with a mean age of $48.6 \pm 23.2$ years old (range: 9-96 years). Cirrhosis was excluded on the basis of the absence of causes for chronic liver disease, with normal results from liver function tests (Child-Pugh classification A). 9 patients $(56.3 \%)$ had an identifiable risk factor (myeloproliferative disorder in 3, antithrombin III deficiency in 2, and antiphospholipid antibody syndrome, protein C deficiency, nephrotic syndrome, and pancreatitis in 1 patient each). All patients had complete venous occlusions involving both the portal veins (PV) and mesenteric veins (MV). 3 patients had occlusions localized at the confluent junction of the main portal vein (MPV) and superior mesenteric vein (SMV); 4 patients had thrombus extension to the intrahepatic PV and segmental MV branches; and 8 patients had simultaneous thrombus involvement from the peripheral venules of the intrahepatic PV to the peripheral MV close to the venous arcade. In the remaining patient, the peripheral venules of the intrahepatic PV were involved, but the peripheral mesenteric venules were spared.

In 9 patients, the time from clinical presentation to treatment was less than 2 weeks; in 3 patients, less than 4 weeks; and in the remaining 4 patients, longer than 4 weeks. 8 patients developed periportal collaterals (cavernoma) on computed tomography (CT) images. Of these 8,4 patients had initial subclinical PMVT symptoms with acute exacerbation.

8 patients had ascites, whereas bowel wall thickening on the CT images was seen in 15 patients and bowel wall thinning in 1 patient, indicating impending bowel ischemia. The main presenting clinical symptoms included abdominal pain in 11 patients, hematemesis in 2 patients, tarry stool in 2 patients, and fever of unknown origin in 1 patient. Interventional treatment was indicated after surgical consultation. Patient demographic data are summarized in Table 1.

Patients were excluded if they showed evidence of liver cirrhosis or malignancy on initial cross-sectional imaging or a contraindication to thrombolysis, including major surgery within the preceding 2 weeks, current active bleeding, allergic reaction to urokinase, fibrinogen under $150 \mathrm{mg} / \mathrm{dL}$ (normal range: $200-400 \mathrm{mg} / \mathrm{dL}$ ) or platelet count under $50,000 / \mathrm{mL}$.

This study was conducted in accordance with the Declaration of Helsinki. All patients gave informed consent before the procedure, and approval from the Institute of the Review Board of Kaohsiung Veterans General Hospital was obtained for this retrospective study.

\section{Thrombolysis technique}

Under sonographic guidance, transhepatic needle puncture of the intrahepatic PV was performed. For the left portal approach, the umbilical segment (either patent or thrombosed) of the left PV was the entry target for puncture by an 18-gauge, $20 \mathrm{~cm}$ needle. For the right portal approach, the patent peripheral PV was punctured with a 21-gauge fine needle, or the thrombosed right segmental PV was punctured with an18-gauge needle if the peripheral portal venules were also thrombosed. A 0.035-inch hydrophilic guidewire (Terumo, Tokyo, Japan) and 4F angio-catheter (RC1, Cordis, CA; J-curve, Terumo) was used to navigate through the occluded venous segments into the patent mesenteric vein.

For patients with localized PMVT, balloon-expandable bare-metal stents (BMS) were directly deployed via a 6-F, 25cm vascular sheath (Terumo, Tokyo) and followed by a short-term urokinase infusion (30,000 IU/hour), if necessary.

For patients with extensive PMVT, 240,000 IU urokinase (diluted with $10 \mathrm{ml}$ normal saline) was initially injected with a $1 \mathrm{cc}$ syringe through the angiocatheter along the thrombosed main PMV (0.5 cc for each forceful injection). After 5-10 minutes' urokinase retention in the thrombosed vessel, catheter aspiration (6F-Envoy, Codman, Johnson \& Johnson or 7F-Mach I, Boston Scientific) and balloon dilation (6-8mm in diameter) were performed. Afterwards, a continuous urokinase infusion (30-60,000 IU/hour concomitant with heparin 300-400 IU/hour) was given via an infusion catheter (Multi-Sideport infusion catheter, Cook, Bloomington, IN; Fountain, Merit, Utah) in the ordinary ward. Angiographic follow-up evaluations with catheter thrombus aspirations and balloon dilation were repeated every 2-3 days. If the peripheral mesenteric venules were involved, an intra-arterial urokinase infusion (30,000 IU/hour) via the superior mesenteric artery (SMA) was given 3-5 days after transhepatic thrombolysis. Bare metal stents (BMS) of $6-10 \mathrm{~mm}$ in diameter were deployed for recurrent/residual stenosis. TIPS creation was considered in order to decrease the peripheral resistance and increase the outflow volume in patients with poor recanalization of the intrahepatic 
portal flow after 7-10 days transhepatic porto-mesenteric thrombolysis. A $10 \mathrm{~mm}$-diameter BMS was used for TIPS establishment. Continuous CDT was performed using an infusion or a conventional catheter until improvement was satisfactory. Intensive monitoring was not necessary during the treatment by this thrombolytic regimen.

Systemic heparinization (3000 IU) and prophylactic antibiotics (cephalexin, 500mg) were given after successful recanalization into the distal patent MV. The patients' coagulation status, including international normalized ration (INR), active partial thromboplastin time (aPTT) and fibrinogen level, was checked before the procedure and every 2-3 days during thrombolysis. If the fibrinogen level fell below $150 \mathrm{mg} / \mathrm{dL}$, it was checked every day. Fresh frozen plasma transfusion was indicated if the fibrinogen level dropped to less than $100 \mathrm{mg} / \mathrm{dL}$. Anticoagulation therapy for at least 6 months was prescribed after discharge.

The degree of clot lysis after treatment was divided into partial recanalization (PR) or complete recanalization (CR). CR represented greater than $90 \%$ clot removal in the mesenteric veins and main portal vein. The degree of lysis in each case was determined in a nonblinded fashion with use of imaging studies, primarily direct and indirect portography by a single interventionalist. Complications were defined as "major" if the duration of hospital stay was extended or treatment was required. Portal vein pressures or portosystemic gradients were not systematically measured in this study.

\section{Statistical analysis}

Continuous variables are presented as median plus range, or as mean \pm 1 standard deviation. Categorical items were expressed as the total value and percentage (\%). Pairs were compared utilizing the Wilcoxon-signed rank test. Nominal variables are given as counts and percentages. Primary PV-patency was defined as time from intervention to first PVT recurrence and secondary PVpatency as time from intervention to permanent technical failure. Kaplan-Meier survival curves were used to measure primary and secondary patency rates. A P value of $<0.05$ was considered statistically significant. Statistical analysis was done using commercially available statistical software (version 17.0; SPSS, Chicago, IL, USA).

\section{Results}

The transhepatic route was adopted in all patients, with a right portal approach in 10 patients and a left portal approach in 6 patients. Additional mesenteric arterial route for indirect thrombolytic infusion was performed in 8 patients. TIPS was created in 3 patients, of which 2 were of the afore-mentioned 8 with additional mesenteric arterial infusions.

Of the 3 localized PMVT patients (Patients 1, 8 and 15), direct BMS (7-10mm in diameter) were deployed with additional urokinase infusions ( 0.4 and 1.2 million IU urokinase in 6 and 20 hours apiece) in 2 patients. Complete recanalization (CR) was noted in all 3 patients on the complete venograms. Patient 8, a 96-year-old patient, died in his sleep 1.5 months after the procedure. Patient 1 was lost to follow-up 4 months after his discharge. Patient 15's stent was patent on his 8-month CT follow-up.

5 patients (Patients 2, 5, 6, 11 and 16) without peripheral mesenteric venules involvement underwent continuous urokinase infusion (mean:7.1 \pm 4.3 million IU, range: 2.4-12.9 million IU), repeated balloon dilation, catheter thrombus aspiration and BMS (7$10 \mathrm{~mm}$ in diameter) placement for 3-15 days' treatment duration. CR (Fig. 1) was achieved in all the 5 patients with TIPS creation at the 10th procedure day to lower the outflow resistance in 1 patient (Patient 16) (Fig. 2). Patient 5 had recurrent gastric variceal bleeding 8 months after the procedure and underwent balloon dilation of the vascular stenosis in the right intrahepatic portal vein. This patient had recurrent bleeding again at 23 months. She then received endoscopic N-Butyl cyanoacrylate (NBCA) injection. Unfortunately, the sclerosing agent spilled out, resulting in total occlusion of the PMV. The other 4 patients had patency of their PMVs at their 2-128 months imaging follow-up.

The 8 patients with peripheral mesenteric venules involvement received both transhepatic and mesenteric arterial route approaches. One female patient (Patient 7), who suffered intermittent abdominal pain for 2 months, refused further local CDT and was shifted to systemic anticoagulant therapy on the 3rd day. She died of ischemic bowel complications on the 9th day. This patient was excluded from later analysis. The other 7 patients underwent continuous urokinase infusion (mean:11.2 \pm 5.6 million $\mathrm{IU}$, range: 4.5-20.4 million IU), repeated balloon dilation, catheter thrombus aspiration, and BMS (6-10 mm in diameter) placement for 6-30 days' treatment duration (mean:13.0 \pm 9.1 days). CR was achieved in 4 patients (Patients $3,4,10$ and 13; see Figs. 3 and 4) and was free of symptoms for up to 118 months' follow-up. 3 patients had poor recanalization of the intrahepatic portal venules. 
Of them, TIPS creation was performed in 2 patients (Patient 9 at the 7th day and Patient 12 at the 11th day). Although the PMV were angiographically patent in these 2 patients but with slow flow demonstrated, Patient 12 complicated with ischemic bowel and died on the 30th day. Patient 14 refused additional TIPS creation, and although we prolonged the local CDT treatment duration to 30 days, we still failed to recanalize the peripheral intrahepatic portal venules, resulting in the re-thrombosis of the major PVs. Although no ischemic bowel was complicated, this patient still complained of intermittent abdominal pain clinically after discharge.

The overall technical success of recanalization in the 15 patients with full courses of treatments was $100 \%$ with CR in 12 patients $(80.0 \%)$ and PR in 3 patients (20.0\%). The 30-day mortality rate was $6.7 \%$ (1 patient). Of the 3 PR patients, one patient (Patient 12) died on the 30th day, and the remaining 2 patients showed re-occlusion of the MPV but with patency of the MV (Patient 9 and 14). As for the $12 \mathrm{CR}$ patients, one 95-year-old patient died at 1.5 months after the procedure without imaging follow-up, one patient complicated with total occlusion of the portal vein after her endoscopic NBCA injection, the other 10 patients present free of symptoms for 4-150 months' clinical follow-up (median: 64.5 months). The treatment techniques and clinical outcomes of these 16 patients are listed in Table 2. Excluding the 2 patients without follow-up images and 1 patient refused further local CDT, the overall 1- and 2-year primary and secondary patency of the PMVT in the 13 patients with 1-128 months (median: 23 months) imaging follow-up were $74.0 \%$ and $84.6 \%$, and $74.0 \%$ and $84.6 \%$, respectively (Fig. 5).

\section{Discussion}

PMVT was previously thought to have an incidence of 1.8 persons per 100,000, according to autopsy studies such as Acosta et $a^{\prime} s^{16}$. With improvements in diagnostic modalities, the incidence rate has been upgraded to 2.7 persons per $100,000{ }^{17}$. Various etiological factors have now been identified in relation to PMVT. The most common etiology includes malignancy (27\%) and cirrhosis (24\%) ${ }^{18}$. Myeloproliferative disorders (MPD), leukemia/lymphoma, inflammatory bowel disease, pancreatitis, postoperative issues (e.g., post-liver transplant, post-splenectomy), sepsis, connective tissue disorders, and various thrombophilic disorders are also common risk factors for PMVT ${ }^{5,18}$. Amongst the thrombophilic states, primary MPD's are the most common, in $30.5 \%{ }^{19}$. Thrombosis of the larger distal portions of the mesenteric vein is mostly secondary to local factors, such as malignancy, pancreatitis and infection, while thrombosis that originates from the vena recta is most-commonly related to a prothrombotic state $^{3}$. Infarction of the bowel mostly requires involvement of the venous arcades and vasa recta ${ }^{17,20}$.

Current guidelines recommend early anticoagulation for at least six months as the standard treatment for acute PMVT ${ }^{5,6}$. Following early anticoagulation, recanalization occurs in $35-45.4 \%{ }^{21}$. In the EN-Vie prospective study, wherein 102 patients were enrolled, the 1 -year recanalization rates were $38 \%$ and $61 \%$ for the portal and superior mesenteric veins, respectively ${ }^{22}$. However, complete recanalization was less frequent and was only achieved in $20 \%$ of patients (19/95) with cavernoma development in $19.9--40 \%{ }^{21,22}$. Furthermore, thrombus resolution is rare in patients with higher degrees of PMVT, ascites, and the presence of more than one prothrombotic risk factor ${ }^{5,22-24}$. In such an emergency situation, local CDT was done with success in some smallseries studies, either through the transjugular route (with or without TIPS creation), a percutaneous transhepatic or transsplenic route, or a transileocolic venous route (either intraoperatively or indirectly via the superior mesenteric artery) ${ }^{7-10,13,25-28}$. In a retrospective comparative study of 34 acute PMVT non-cirrhotic patients, Liu et al concluded that indirect thrombolysis via the SMA was safer and more effective than systemic thrombolysis (ST) ${ }^{28}$. Another paper reported that the recanalization effect of direct thrombolysis is better than that of indirect treatment (14.3\% partial recanalization) ${ }^{10}$. Hollingshead et a/ recommended a combined direct and indirect thrombolysis simultaneously for more extensive PMVT patents ${ }^{9}$. In this study, we started the adjunctive mesenteric arterial thrombolytic infusion in PMVT patients with peripheral mesenteric venules involvement at 3-5 days after the transhepatic treatment with partial recanalization achieved, in order to avoid high concentrations of urokinase retention in the intestinal mucosa, which might carry higher potential risk of intestinal bleeding and also to shorten the femoral arterial indwelling time and patients' discomfort.

Smalberg et al ${ }^{15}$ and Hollingshead et al ${ }^{9}$ reported major-procedure-related bleeding complications in $50 \%$ and $60 \%$ respectively of patients treated with local intravascular thrombolysis via the transhepatic approach and/or indirect thrombolysis via the SMA ${ }^{9}$. Thereafter, the transhepatic approach was regarded as unsafe, and the transjugular approach (with or without TIPS creation) 
became the mainstream approach for direct local thrombolysis ${ }^{9,29}$. It is worth noting that the dosages of thrombolytic agents used in these two studies (100,000-240,000 IU/hour urokinase concomitant with 1250 IU/hour heparin in Hollingshead's series ${ }^{9}$ and rtPA of 2-4 mg/hour in Smalberg's series $\left.{ }^{15}\right)$ were much higher than those of other series (60.000 IU urokinase or $1 \mathrm{mg}$ rtPA per hour rtPA) $7,10,13$ and the present study $(10,000-60,000 \mathrm{IU} /$ hour urokinase with 300-400 IU/hour). The different dosages could themselves explain the different bleeding complicating rates, rather than the different approach routes. Even in Rabuffi series ${ }^{14}$, they adopted the transhepatic route to treat 3 acute PMVT patients by a 10-Fr atherectomy device and followed by infusion of 80,000-100,000 IU urokinase per hour without bleeding complication encountered. Accordingly, we can conclude that the major bleeding complication of the thrombolytic therapy is more dose-related other than the transhepatic route approach.

The rationale of creating a low pressure system in TIPS is to act as a vacuum for clot fragments and improve the effectiveness of thrombolysis in managing PMVT ${ }^{30}$. In theory, TIPS creation in the non-cirrhotic patients is not physiological. Moreover, it is more difficult to create TIPS in the presence of portal vein thrombosis. For the safety of ensuing thrombolytic therapy, techniques with fewer needle puncture passes should be adopted. Surveying previous studies shows that the technical success rate of TIPS in patients with PMVT can be significantly variable, ranging from 35-83\%11,25,26,30-34. Qi et al reported that TIPS was successfully placed in $35 \%(7 / 20)$ of their patients with MPV thrombosis, via a transjugular approach alone in 1 successful patient (5\%) and a combined transjugular and transhepatic or transsplenic approach in the other 6 successful patients ${ }^{26}$. Chen et a/ proposed a transhepatic balloon-assisted technique to facilitate TIPS creation in the MPV occluded cirrhotic patients and reported $77.8 \%$ technical success with exclusion of mesenteric venous occlusion ${ }^{33}$. Fanelli et al used a thin $(21 \mathrm{G})$ TIPS-set needle with multiple blind punctures under fluoroscopic guidance with technical success of $83 \%{ }^{25}$. In Luo et als series, TIPS placement was technically successful in $73.3 \%$ (11/15) of patients, and all successful cases had either a patent SMV or a large porto-porto collateral vein ${ }^{11}$. The conclusions of such studies were that TIPS was not recommended in patients with a total fibrotic cord and/or without a large collateral vein or in those with extensive superior mesenteric vein thrombosis ${ }^{34}$. Benmassaoud et al reported two major complications of subcapsular hematoma and hepatic encephalopathy during or after TIPS creation, out of a total of 22 patients ${ }^{7}$. In our series, $100 \%$ technical success of the transhepatic approach was achieved, without any procedurerelated major bleeding complications. Meanwhile, although patients with cirrhosis were excluded from the present study, none were excluded from this study due to thrombus extension, fibrotic cord of MPV or cavernoma formation.

Portal vein thrombosis is classified based on the clinical and imaging presentation (acute or subacute vs chronic; benign vs malignant, and intrahepatic vs extrahepatic extent). These classifications guide therapy and have prognostic implications ${ }^{29}$. Although current guidelines recommend early anticoagulation as the standard treatment for acute PMVT 5,6, the definition of acute PMVT is ambiguous. Parikh et al ${ }^{35}$ considered acute PMVT as symptoms develop $<60$ days before presentation, and there is no clinical, radiological, or endoscopic evidence of portal hypertension or collateral circulation. Singal et al ${ }^{36}$ stated that acute MVT is characterized by symptomatic presentation within 24 to 72 hours of thrombus formation with sudden onset of symptoms, whereas subacute MVT presents during days to weeks of nonspecific symptoms. Chronic PMVT usually presents with complications of portal hypertension or may be detected as an incidental finding on computed tomography (CT) imaging (28.1\%) 23,36. As the symptoms of chronic PMVT may be acutely exacerbated due to thrombus progression into the peripheral mesenteric veins, it is imperative to reestablish porto-mesenteric blood flow as soon as possible. Benmassaoud et a/proposed a stepwise protocol by using systemic anticoagulation or thrombolysis first and then followed by local CDT for treatment-resistant patients ${ }^{7}$. In their series, the average duration before initiation of CDT was 4 days, stating that longer intervals would be associated with a decreased chance of recanalization, leaving the patient at risk of progressive intestinal ischemia and long-term sequelae of portal hypertension. As complete recanalization from systemic therapy was only achieved of $13.6 \%(3 / 22)$ of their patients ${ }^{7}$, and our protocol of transhepatic CDT under ultrasonographic guidance puncture with tailored thrombolytic regimen carries few risks, it is our recommendation that primary local CDT should be started as soon as possible once the diagnosis of PMVT is made, even under the circumstance that the thrombus might be chronic. Two previous articles, focusing on managing the chronic non-cirrhotic portal venous thrombosis via TIPS and metallic stenting without infusions of thrombolytic agents ${ }^{11,26}$, reported the technical success of $35 \%$ in one series ${ }^{26}$ and $50 \%$ in the other series if the mesenteric vein were also involved. To our best knowledge, the present study reports the first experience of managing including the chronic PMVT by local CDT with a tailored dosage (e.g. 240,000 IU urokinase for pulse-spray injection and 60,000-30,000 IU per hour for even up to 30 days' continuous infusion) without 
the need of intensive monitoring. The experience of clinical success to dissolve chronic thrombus is similar to some previous reports on chronic arterial occlusive disease ${ }^{37}$ and chronic PTFE graft occlusion in hemodialysis patients ${ }^{38}$.

Previous studies have shown recanalization rates of CDT for PMVT ranging from 52.3-94.1\%, with complete recanalization of $6.3-52.9 \% 9,13,39-42$. Klinger et al reported that the one- and two-year secondary PV patency rates in 17 consecutive acute noncirrhotic PMVT patients were both $88.2 \%$ without significant difference in patients with TIPS-implantation at the end of thrombolysis compared to patients without TIPS-implantation $(p=0.2)^{13}$. In our series, the overall successful recanalization rate of the PMVT patients was $100 \%$ (15 patients) with complete recanalization in $80.0 \%$ (12/15 patients). The 2 patients with partial recanalization even after TIPS creation eventually showed re-occlusion of the PMV. All PMVT patients in the present study with complete recanalization showed good long-term patency. As a result, completely recanalizing the thrombosed PMV should be the goal of this treatment strategy with TIPS creation only for recanalization failure of the intra-hepatic portal flow in these noncirrhotic PMVT patients. The overall one- and two-year secondary patency rates in our series were both $84.6 \%$.

\section{Limitations}

Firstly, our study was limited due to the retrospective, non-controlled study design and a small study population of the noncirrhotic PMVT. Unfortunately, this is the reality when studying a rare disease associated with significant morbidity, mortality, and limited therapeutic options. Secondly, thrombectomy devices were not used to accelerate the clearance of acute or subacute thrombi in our study, as they are expensive and a clinically acute presentation may be actually be caused by a chronic thrombus with acute clinical exacerbation. The real clinical benefits of the thrombectomy devices in these patients may be limited. Lastly, the long-term vascular patency, especially of the partially recanalized PMV, cannot be determined from such a small patient group.

\section{Conclusion}

Acute clinical presentations of PMVT do not reflect the actual thrombus stage. Local CDT can be performed as a primary salvage treatment once the diagnosis is made. The goal of treatment strategy for PMVT should be to achieve complete recanalization. CDT via the transhepatic route with a tailored thrombolytic regimen is safe and effective in both acute and chronic PMVT. TIPS creation can be preserved for non-cirrhotic PMVT patients only if recanalization failure of the intrahepatic PVs.

\section{References}

1. Grendell, J. H. \& Ockner, R. K. Mesenteric venous thrombosis., 82, 358-372 (1982).

2. Søgaard, K. K., Darvalics, B., Horváth-Puhó, E. \& Sørensen, H. T. Survival after splanchnic vein thrombosis: A 20-year nationwide cohort study. Thrombosis research, 141, 1-7 https://doi.org/10.1016/j.thromres.2016.02.024 (2016).

3. Hmoud, B., Singal, A. K. \& Kamath, P. S. Mesenteric venous thrombosis. J Clin Exp Hepatol, 4, 257-263 https://doi.org/10.1016/j.jceh.2014.03.052 (2014).

4. Chawla, Y. K. \& Bodh, V. Portal vein thrombosis. J Clin Exp Hepatol, 5, 22-40 https://doi.org/10.1016/j.jceh.2014.12.008 (2015).

5. European Association for the Study of the Liver. Electronic address, e. e. e. EASL Clinical Practice Guidelines: Vascular diseases of the liver. J Hepatol, 64, 179-202 https://doi.org/10.1016/j.jhep.2015.07.040 (2016).

6. DeLeve, L. D., Valla, D. C. \& Garcia-Tsao, G. \& American Association for the Study Liver, D. Vascular disorders of the liver., 49, 1729-1764 https://doi.org/10.1002/hep.22772 (2009).

7. Benmassaoud, A. et al. A stepwise thrombolysis regimen in the management of acute portal vein thrombosis in patients with evidence of intestinal ischaemia. Aliment Pharmacol Ther, 50, 1049-1058 https://doi.org/10.1111/apt.15479 (2019).

8. Bilbao, J. I. et al. Transjugular intrahepatic portocaval shunt after thrombus disruption in partially thrombosed portal veins. Cardiovascular and interventional radiology, 17, 106-109 https://doi.org/10.1007/BF00193927 (1994).

9. Hollingshead, M. et al. Transcatheter thrombolytic therapy for acute mesenteric and portal vein thrombosis. Journal of vascular and interventional radiology: JVIR, 16, 651-661 https://doi.org/10.1097/01.rvi.0000156265.79960.86 (2005). 
10. Liu, F. Y. et al. Interventional treatment for symptomatic acute-subacute portal and superior mesenteric vein thrombosis. World journal of gastroenterology, 15, 5028-5034 https://doi.org/10.3748/wjg.15.5028 (2009).

11. Luo, X. et al. Transjugular intrahepatic portosystemic shunt for the treatment of portal hypertension in noncirrhotic patients with portal cavernoma. Gastroenterology research and practice, 2014, https://doi.org/10.1155/2014/659726 (2014).

12. Rosenqvist, K., Ebeling Barbier, C., Rorsman, F., Sangfelt, P. \& Nyman, R. Treatment of acute portomesenteric venous thrombosis with thrombectomy through a transjugular intrahepatic portosystemic shunt: a single-center experience. Acta radiologica (Stockholm, Sweden: 1987) 59, 953-958, doi:10.1177/0284185117742683 (2018).

13. Klinger, C. et al. Transjugular local thrombolysis with/without TIPS in patients with acute non-cirrhotic, non-malignant portal vein thrombosis. Digestive and liver disease: official journal of the Italian Society of Gastroenterology and the Italian Association for the Study of the Liver, 49, 1345-1352 https://doi.org/10.1016/j.dld.2017.05.020 (2017).

14. Rabuffi, P., Vagnarelli, S., Bruni, A., Antonuccio, G. \& Ambrogi, C. Percutaneous Pharmaco-Mechanical Thrombectomy of Acute Symptomatic Superior Mesenteric Vein Thrombosis. Cardiovascular and interventional radiology, 43, 46-54 https://doi.org/10.1007/s00270-019-02354-y (2020).

15. Smalberg, J. H. et al. Risks and benefits of transcatheter thrombolytic therapy in patients with splanchnic venous thrombosis. Thrombosis and haemostasis, 100, 1084-1088 (2008).

16. Acosta, S., Alhadad, A., Svensson, P. \& Ekberg, O. Epidemiology, risk and prognostic factors in mesenteric venous thrombosis. The British journal of surgery, 95, 1245-1251 https://doi.org/10.1002/bjs.6319 (2008).

17. Harnik, I. G. \& Brandt, L. J. Mesenteric venous thrombosis. Vascular medicine (London, England), 15, 407-418 https://doi.org/10.1177/1358863×10379673 (2010).

18. Thatipelli, M. R., McBane, R. D., Hodge, D. O. \& Wysokinski, W. E. Survival and recurrence in patients with splanchnic vein thromboses. Clinical gastroenterology and hepatology: the official clinical practice journal of the American Gastroenterological Association, 8, 200-205 https://doi.org/10.1016/j.cgh.2009.09.019 (2010).

19. Condat, B. \& Valla, D. Nonmalignant portal vein thrombosis in adults. Nature clinical practice. Gastroenterology \& hepatology, 3, 505-515 https://doi.org/10.1038/ncpgasthep0577 (2006).

20. Kumar, S., Sarr, M. G. \& Kamath, P. S. Mesenteric venous thrombosis. The New England journal of medicine, 345, 1683-1688 https://doi.org/10.1056/NEJMra010076 (2001).

21. Hall, T. C., Garcea, G., Metcalfe, M., Bilku, D. \& Dennison, A. R. Management of acute non-cirrhotic and non-malignant portal vein thrombosis: a systematic review. World journal of surgery, 35, 2510-2520 https://doi.org/10.1007/s00268-011-1198-0 (2011).

22. Plessier, A. et al. Acute portal vein thrombosis unrelated to cirrhosis: a prospective multicenter follow-up study., 51, 210-218 https://doi.org/10.1002/hep.23259 (2010).

23. Amitrano, L. et al. Prognostic factors in noncirrhotic patients with splanchnic vein thromboses. The American journal of gastroenterology, 102, 2464-2470 https://doi.org/10.1111/j.1572-0241.2007.01477.x (2007).

24. Turnes, J. et al. Portal hypertension-related complications after acute portal vein thrombosis: impact of early anticoagulation. Clinical gastroenterology and hepatology: the official clinical practice journal of the American Gastroenterological Association, 6, 1412-1417 https://doi.org/10.1016/j.cgh.2008.07.031 (2008).

25. Fanelli, F. et al. Transjugular intrahepatic portosystemic shunt with expanded-polytetrafuoroethylene-covered stents in noncirrhotic patients with portal cavernoma. Digestive and liver disease: official journal of the Italian Society of Gastroenterology and the Italian Association for the Study of the Liver, 43, 78-84 https://doi.org/10.1016/j.dld.2010.06.001 (2011).

26. Qi, X. et al. Transjugular intrahepatic portosystemic shunt for portal cavernoma with symptomatic portal hypertension in noncirrhotic patients. Digestive diseases and sciences, 57, 1072-1082 https://doi.org/10.1007/s10620-011-1975-5 (2012).

27. Fonseca, A. L., Cleary, M. A., Cholewczynski, W., Sumpio, B. E. \& Atweh, N. A. Omental vein catheter thrombolysis for acute porto-mesenteric vein thrombosis. Annals of vascular surgery, 27, 497491-497494 https://doi.org/10.1016/j.avsg.2012.09.009 (2013).

28. Liu, K., Li, W. D., Du, X. L., Li, C. L. \& Li, X. Q. Comparison of Systemic Thrombolysis Versus Indirect Thrombolysis via the Superior Mesenteric Artery in Patients with Acute Portal Vein Thrombosis. Annals of vascular surgery, 39, 264-269

Page $8 / 14$ 
https://doi.org/10.1016/j.avsg.2016.06.029 (2017).

29. Chamarthy, M. R., Anderson, M. E., Pillai, A. K. \& Kalva, S. P. Thrombolysis and Transjugular Intrahepatic Portosystemic Shunt Creation for Acute and Subacute Portal Vein Thrombosis. Techniques in vascular and interventional radiology, 19, 42-51 https://doi.org/10.1053/j.tvir.2016.01.005 (2016).

30. Semiz-Oysu, A., Keussen, I. \& Cwikiel, W. Interventional radiological management of prehepatic obstruction of [corrected] the splanchnic venous system. Cardiovascular and interventional radiology, 30, 688-695 https://doi.org/10.1007/s00270-0079097-3 (2007).

31. Senzolo, M. et al. Transjugular intrahepatic portosystemic shunt for portal vein thrombosis with and without cavernous transformation. Aliment Pharmacol Ther, 23, 767-775 https://doi.org/10.1111/j.1365-2036.2006.02820.x (2006).

32. Perarnau, J. M., Baju, A., D'Alteroche, L., Viguier, J. \& Ayoub, J. Feasibility and long-term evolution of TIPS in cirrhotic patients with portal thrombosis. European journal of gastroenterology \& hepatology, 22, 1093-1098 https://doi.org/10.1097/MEG.0b013e328338d995 (2010).

33. Chen, Y. et al. Percutaneous transhepatic balloon-assisted transjugular intrahepatic portosystemic shunt for chronic, totally occluded, portal vein thrombosis with symptomatic portal hypertension: procedure technique, safety, and clinical applications. European radiology, 25, 3431-3437 https://doi.org/10.1007/s00330-015-3777-1 (2015).

34. Qi, X. \& Han, G. Transjugular intrahepatic portosystemic shunt in the treatment of portal vein thrombosis: a critical review of literature. Hepatology international, 6, 576-590 https://doi.org/10.1007/s12072-011-9324-5 (2012).

35. Parikh, S., Shah, R. \& Kapoor, P. Portal vein thrombosis. The American journal of medicine, 123, 111-119 https://doi.org/10.1016/j.amjmed.2009.05.023 (2010).

36. Singal, A. K., Kamath, P. S. \& Tefferi, A. Mesenteric venous thrombosis. Mayo Clinic proceedings 88, 285-294, doi:10.1016/j.mayocp.2013.01.012 (2013).

37. Liang, H. L., Li, M. F., Hsiao, C. C., Wu, C. J. \& Wu, T. H. Endovascular management of aorto-iliac occlusive disease (Leriche syndrome). Journal of the Formosan Medical Association = Taiwan yi zhi, https://doi.org/10.1016/j.jfma.2020.10.033 (2020).

38. Weng, M. J. et al. Endovascular revascularization of chronically thrombosed arteriovenous fistulas and grafts for hemodialysis: a retrospective study in 15 patients with 18 access sites. Cardiovascular and interventional radiology, 34, 319$330 \mathrm{https} / / /$ doi.org/10.1007/s00270-010-9926-7 (2011).

39. Lopera, J. E. et al. Percutaneous transhepatic treatment of symptomatic mesenteric venous thrombosis. Journal of vascular surgery, 36, 1058-1061 https://doi.org/10.1067/mva.2002.127526 (2002).

40. Aytekin, C., Boyvat, F., Kurt, A., Yologlu, Z. \& Coskun, M. Catheter-directed thrombolysis with transjugular access in portal vein thrombosis secondary to pancreatitis. European journal of radiology, 39, 80-82 https://doi.org/10.1016/s0720048x(01)00289-3 (2001).

41. Kercher, K. W. et al. Transhepatic thrombolysis in acute portal vein thrombosis after laparoscopic splenectomy. Surgical laparoscopy, endoscopy \& percutaneous techniques, 12, 131-136 https://doi.org/10.1097/00129689-200204000-00013 (2002).

42. Schoots, I. G., Koffeman, G. I., Legemate, D. A., Levi, M. \& van Gulik, T. M. Systematic review of survival after acute mesenteric ischaemia according to disease aetiology. The British journal of surgery, 91, 17-27 https://doi.org/10.1002/bjs.4459 (2004).

\section{Tables}

Table1. Demographic data of the 16 PMVT patients 


\begin{tabular}{|c|c|c|c|c|c|c|c|c|c|c|}
\hline No & Sex & Age & symptoms & onset & etiology & $a / c$ & cavernoma & $\begin{array}{l}\text { wall } \\
\text { edema }\end{array}$ & ascites & $\begin{array}{l}\text { peripheral } \\
\text { venules }\end{array}$ \\
\hline 1 & $M$ & 69 & $\begin{array}{l}\text { abdominal } \\
\text { pain }\end{array}$ & 3 days & unknown & acute & nil & $y$ & nil & nil \\
\hline 2 & $F$ & 9 & $\begin{array}{l}\text { abdominal } \\
\text { pain }\end{array}$ & $\begin{array}{l}6 \\
\text { months }\end{array}$ & $\begin{array}{l}\text { nephrotic } \\
\text { syndrome }\end{array}$ & chronic & $y$ & $y$ & $y$ & nil \\
\hline 3 & M & 37 & $\begin{array}{l}\text { abdominal } \\
\text { pain }\end{array}$ & 7 days & $\begin{array}{l}\text { antiphospholipid } \\
\text { antibody } \\
\text { syndrome }\end{array}$ & acute & nil & $y$ & nil & $P V+M V$ \\
\hline 4 & $M$ & 47 & $\begin{array}{l}\text { abdominal } \\
\text { pain }\end{array}$ & $\begin{array}{l}14 \\
\text { days }\end{array}$ & unknown & acute & nil & $y$ & nil & MV \\
\hline 5 & $F$ & 11 & $\begin{array}{l}\text { variceal } \\
\text { bleeding }\end{array}$ & 3 years & $\begin{array}{l}\text { protein C } \\
\text { deficiency }\end{array}$ & chronic & $y$ & $y$ & $y$ & nil \\
\hline 6 & $M$ & 26 & $\begin{array}{l}\text { variceal } \\
\text { bleeding }\end{array}$ & $\begin{array}{l}4 \\
\text { months }\end{array}$ & $\begin{array}{l}\text { myeloproliferative } \\
\text { disorder }\end{array}$ & chronic & $y$ & $y$ & nil & nil \\
\hline 7 & $\mathrm{~F}$ & 73 & $\begin{array}{l}\text { abdominal } \\
\text { pain }\end{array}$ & $\begin{array}{l}2 \\
\text { months }\end{array}$ & unknown & chronic & $y$ & $y$ & nil & $\mathrm{PV}+\mathrm{MV}$ \\
\hline 8 & $M$ & 96 & tarry stool & $\begin{array}{l}12 \\
\text { days }\end{array}$ & unknown & chronic & nil & $y$ & nil & nil \\
\hline 9 & $M$ & 35 & $\begin{array}{l}\text { abdominal } \\
\text { pain }\end{array}$ & $\begin{array}{l}26 \\
\text { days }\end{array}$ & $\begin{array}{l}\text { myeloproliferative } \\
\text { disorder }\end{array}$ & subacute & $y$ & $y$ & $y$ & $P V+M V$ \\
\hline 10 & $M$ & 40 & $\begin{array}{l}\text { abdominal } \\
\text { pain }\end{array}$ & 3 days & $\begin{array}{l}\text { antithrombin III } \\
\text { deficiency }\end{array}$ & chronic & $y$ & $y$ & $y$ & $P V+M V$ \\
\hline 11 & $M$ & 76 & $\begin{array}{l}\text { fever of } \\
\text { unknown } \\
\text { origin }\end{array}$ & $\begin{array}{l}14 \\
\text { days }\end{array}$ & $\begin{array}{l}\text { antithrombin III } \\
\text { deficiency }\end{array}$ & acute & nil & thinning & nil & PV \\
\hline 12 & $M$ & 49 & $\begin{array}{l}\text { abdominal } \\
\text { pain }\end{array}$ & 2 days & $\begin{array}{l}\text { myeloproliferative } \\
\text { disorder }\end{array}$ & acute & nil & $y$ & $y$ & $P V+M V$ \\
\hline 13 & $M$ & 59 & $\begin{array}{l}\text { abdominal } \\
\text { pain }\end{array}$ & 4 days & unknown & chronic & $y$ & $y$ & $y$ & $P V+M V$ \\
\hline 14 & $\mathrm{~F}$ & 51 & $\begin{array}{l}\text { abdominal } \\
\text { pain }\end{array}$ & $\begin{array}{l}14 \\
\text { days }\end{array}$ & unknown & subacute & nil & $y$ & $y$ & $P V+M V$ \\
\hline 15 & $M$ & 56 & tarry stool & $\begin{array}{l}23 \\
\text { days }\end{array}$ & pancreatitis & chronic & nil & $y$ & $y$ & nil \\
\hline 16 & M & 44 & $\begin{array}{l}\text { abdominal } \\
\text { pain }\end{array}$ & $\begin{array}{l}22 \\
\text { days }\end{array}$ & unknown & chronic & $y$ & $y$ & nil & PV \\
\hline
\end{tabular}

M: male; F: female

Table2. Technical perspectives and clinical outcomes of the 16 PMVT patients 


\begin{tabular}{|c|c|c|c|c|c|c|c|c|c|}
\hline No & route & $\mathrm{L} / \mathrm{R}$ & $\begin{array}{l}\text { lysis } \\
\text { days }\end{array}$ & $\begin{array}{l}\text { UK dose } \\
\text { (million) }\end{array}$ & $\begin{array}{l}\text { BMS size } \\
(\mathrm{mm})\end{array}$ & recanalization & $\begin{array}{l}\text { follow-up } \\
\text { image }\end{array}$ & status & survival \\
\hline 1 & $\mathrm{TH}$ & $\mathrm{R}$ & 0.4 & 0.4 & $7 \times 60$ & CR & CT-1m & patent & $4 m-I f u$ \\
\hline 2 & $\mathrm{TH}$ & $\mathrm{R}$ & 12 & 6.8 & $8 \times 100(2)$ & $\mathrm{CR}$ & US-128m & patent & $150 m$ \\
\hline 3 & $\mathrm{TH}+\mathrm{SMA}$ & L & 9 & 8.6 & $6 \times 120$ & $\mathrm{CR}$ & CT-105m & patent & $119 m$ \\
\hline 4 & TH+SMA & L & 3 & 4.5 & $6 \times 60$ & CR & CT-79m & patent & $110 \mathrm{~m}$ \\
\hline 5 & $\mathrm{TH}$ & $\mathrm{R}$ & 6 & 2.4 & $8 \times 60$ & $\mathrm{CR}$ & US-8m & stenosis & $106 m$ \\
\hline 6 & $\mathrm{TH}$ & $\mathrm{R}$ & 13 & 9.6 & $8 \times 100$ & $\mathrm{CR}$ & CT-26m & patent & $105 m$ \\
\hline 7 & $\mathrm{TH}+\mathrm{SMA}$ & $\mathrm{R}$ & 2 & 2.8 & nil & $\begin{array}{l}\text { refused } \\
\text { further } \mathrm{CDT}\end{array}$ & nil & excluded & $\begin{array}{l}\text { expired-9 } \\
\text { days }\end{array}$ \\
\hline 8 & $\mathrm{TH}$ & $\mathrm{R}$ & 3 & $x$ & $7 \times 60$ & CR & nil & uncertain & $\begin{array}{l}\text { expired- } \\
1.5 \mathrm{~m}\end{array}$ \\
\hline 9 & TH+SMA+TIPS & $\mathrm{R}$ & 19 & 16.4 & $8 \times 80$ & PR & CT-1m & $\begin{array}{l}\text { re- } \\
\text { occluded }\end{array}$ & $2 m-I f u$ \\
\hline 10 & $\mathrm{TH}+\mathrm{SMA}$ & L & 12 & 11.6 & nil & $\mathrm{CR}$ & CT-65m & patent & $68 m$ \\
\hline 11 & $\mathrm{TH}$ & L & 3 & 3.6 & $8 \times 37$ & $\mathrm{CR}$ & US-36m & patent & $41 m$ \\
\hline 12 & TH+IMA+TIPS & $\mathrm{R}$ & 12 & 10.8 & $10 \times 80$ & PR & nil & uncertain & $\begin{array}{l}\text { expired- } \\
30 \text { days }\end{array}$ \\
\hline 13 & $\mathrm{TH}+\mathrm{SMA}$ & L & 6 & 6.4 & nil & $\mathrm{CR}$ & MR-15m & patent & $25 m$ \\
\hline 14 & $\mathrm{TH}+\mathrm{SMA}$ & $\mathrm{R}$ & 30 & 20.4 & $8 \times 40$ & $\begin{array}{l}\text { PR: refused } \\
\text { TIPS }\end{array}$ & CT-1m & $\begin{array}{l}\text { re- } \\
\text { occluded }\end{array}$ & $18 m$ \\
\hline 15 & $\mathrm{TH}$ & $\mathrm{R}$ & 1 & 1.2 & $10 \times 37$ & $\mathrm{CR}$ & CT-5m & patent & $14 \mathrm{~m}$ \\
\hline 16 & TH+TIPS & L & 15 & 12.9 & $8 \times 60$ & CR & CT-2m & patent & $5 m$ \\
\hline
\end{tabular}

TH: transhepatic; SMA: superior mesenteric artery; TIPS: transjugular intrahepatic porto-systemic shunt; R: right; L: left; UK: urokinase

BMS: bare-metal stent; CR: complete recanalization; PR: partial recanalization; CDT: catheter-directed thrombolysis; Ifu: loss to follow-up

\section{Figures}
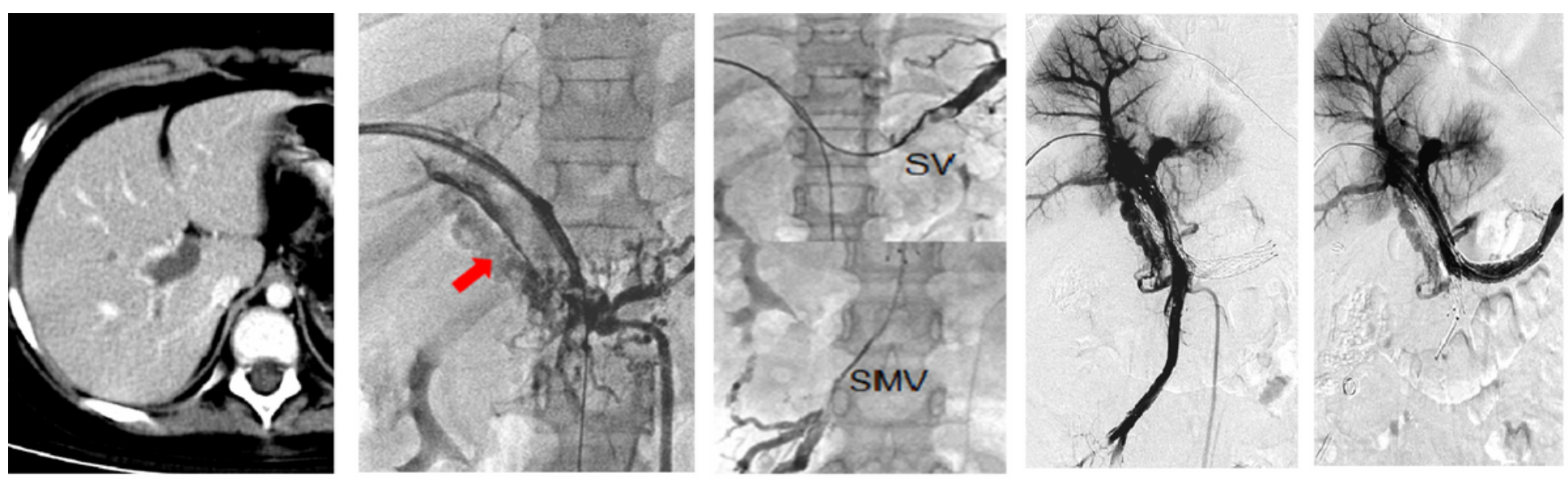

Figure 1 
Chronic PMVT in a 9-year-old girl diagnosed with nephrotic syndrome, with complaints of intermittent abdominal pain for 6 months A. CT image revealed diffuse PMVT B. Transhepatic portogram confirmed CT findings. Arrow: cavernoma C. Upper: splenic vein (SV) thrombosis. Lower: SMV thrombosis. D-E: Transhepatic portograms showed recanalization of the SMV, SV, MPV and intrahepatic portal veins after continuous urokinase infusion (total dose of 6.8 million IU for 12 days' infusion), BMS placement $(8 \times 100 \mathrm{~mm})$ and repeated balloon dilation. This patient had been symptom-free for more than 10 years at the time of writing, with patent vascular flow on the last color Doppler ultrasound follow-up 128 months after the procedure.
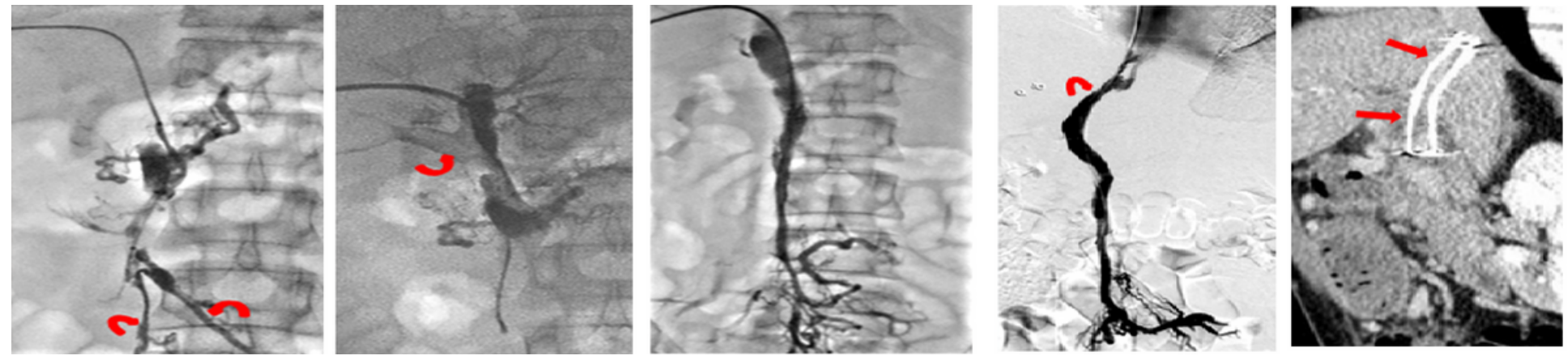

\section{Figure 2}

Chronic PMVT with acute exacerbation in a 44-year-old male patient presenting with abdominal pain for 22 days. A. Transhepatic portogram revealed diffuse PMVT involving the peripheral venules of the intra-hepatic PV, leaving the peripheral mesenteric venules spared (curved arrows). B. Transhepatic portogram demonstrated faint partial opacification of the right portal vein (curved arrow) after continuous urokinase infusion and repeated catheter thrombus aspiration. C. Transhepatic portogram showed although near complete recanalization of the mesenteric veins and the main portal vein, no further thrombus lysis without contrast opacification of the intrahepatic portal thrombus was seen. D. A TIPS shunt (curved arrow) was created on the 10th procedure day to lower the outflow resistance. E. CT images (sagittal oblique reformation) at 3 months' follow-up revealed recanalization of the porto-mesenteric vein with patency of the TIPS shunt (arrows).
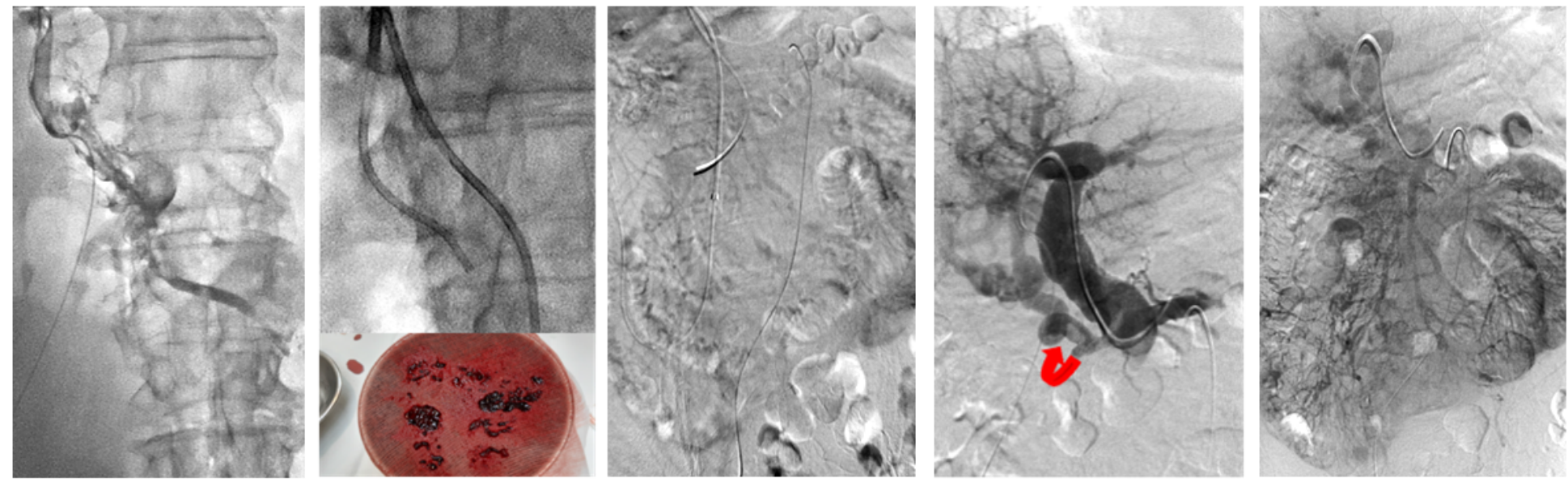

\section{Figure 3}

A 58-year-old male patient with abdominal pain for 4 days. 5 years prior, he underwent segmental ileal resection due to venous occlusive ischemic bowel. A. Transhepatic portogram showed extensive PMVT involving the peripheral venules of both the intrahepatic PV and MV close to the venous arcade. B. Upper: Fluoro-image demonstrated catheter aspiration (6F Envoy catheter) in the thrombosed PMV. Lower: photograph showed some fresh and old blood clots aspirated out. C. SMA angiograms (venous phase) failed to opacify the segmental mesenteric veins. D. Transhepatic portogram showed recanalization of the main and intrahepatic PVs after repeated balloon dilation, catheter aspiration and continuous urokinase infusion via both the transhepatic portal ( 6 days' duration) and transarterial SMA (3 days' duration) routes. Curved arrow: residual cavernoma E. SMA angiograms (venous phase) demonstrated re-opacification of the mesenteric veins and portal veins after treatments. 

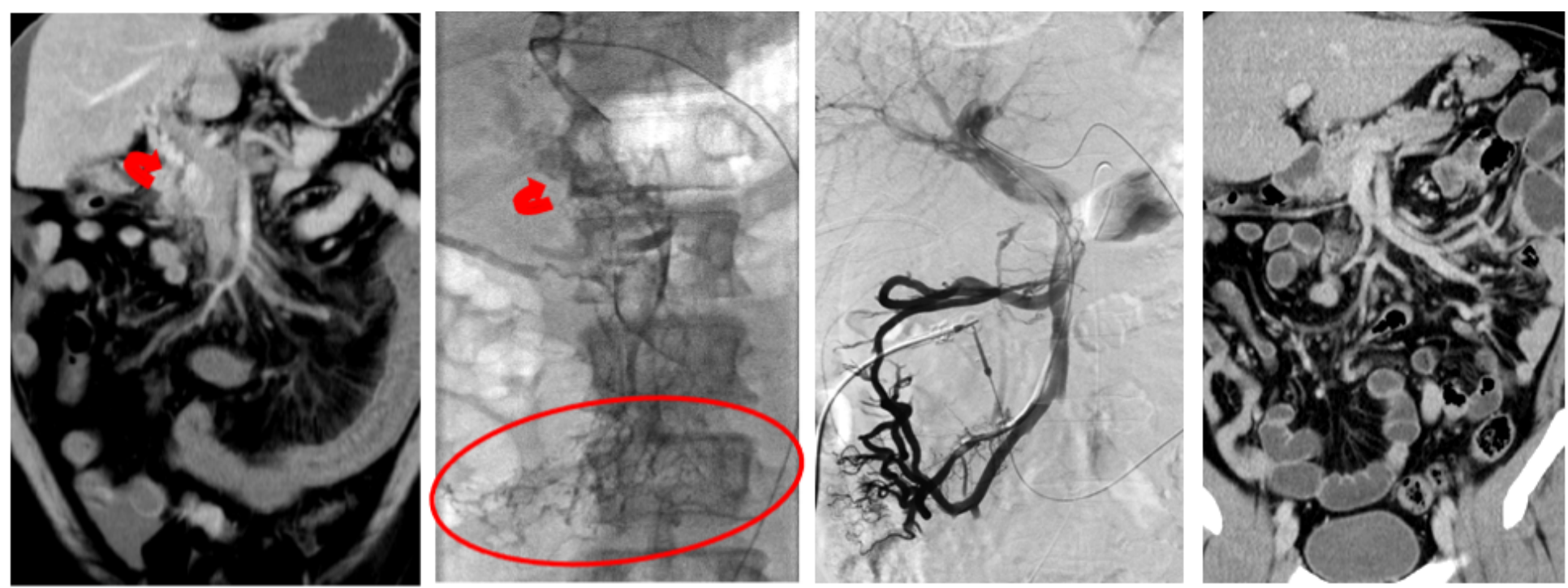

Figure 4

Chronic PMVT with acute exacerbation in a 40-year-old male patient diagnosed as antithrombin III deficiency with complaint of abdominal pain for 3 days. A. Coronal CT image showed total occlusion of the PMV with bowel wall edema and ascites, as noted on this CT image. There was cavernoma formation (curved arrow) in this acute onset chronic PMVT. B. Transhepatic portogram confirmed the CT findings of diffuse thrombosis involving the peripheral mesenteric venules (circle) and major and intra-hepatic portal veins. Curved arrow: cavernoma formation C. Transhepatic portogram revealed recanalization of the mesenteric veins and major and intrahepatic portal veins after local CDT, via both transhepatic and SMA routes, with the total urokinase dose of 11.6 million IU for 12 days' infusion. Non-opacification of the cavernoma was noted on the complete portogram. D. Coronal CT image at 8-month' follow-up revealed patency of the PMV with normal appearance of the bowel loop.

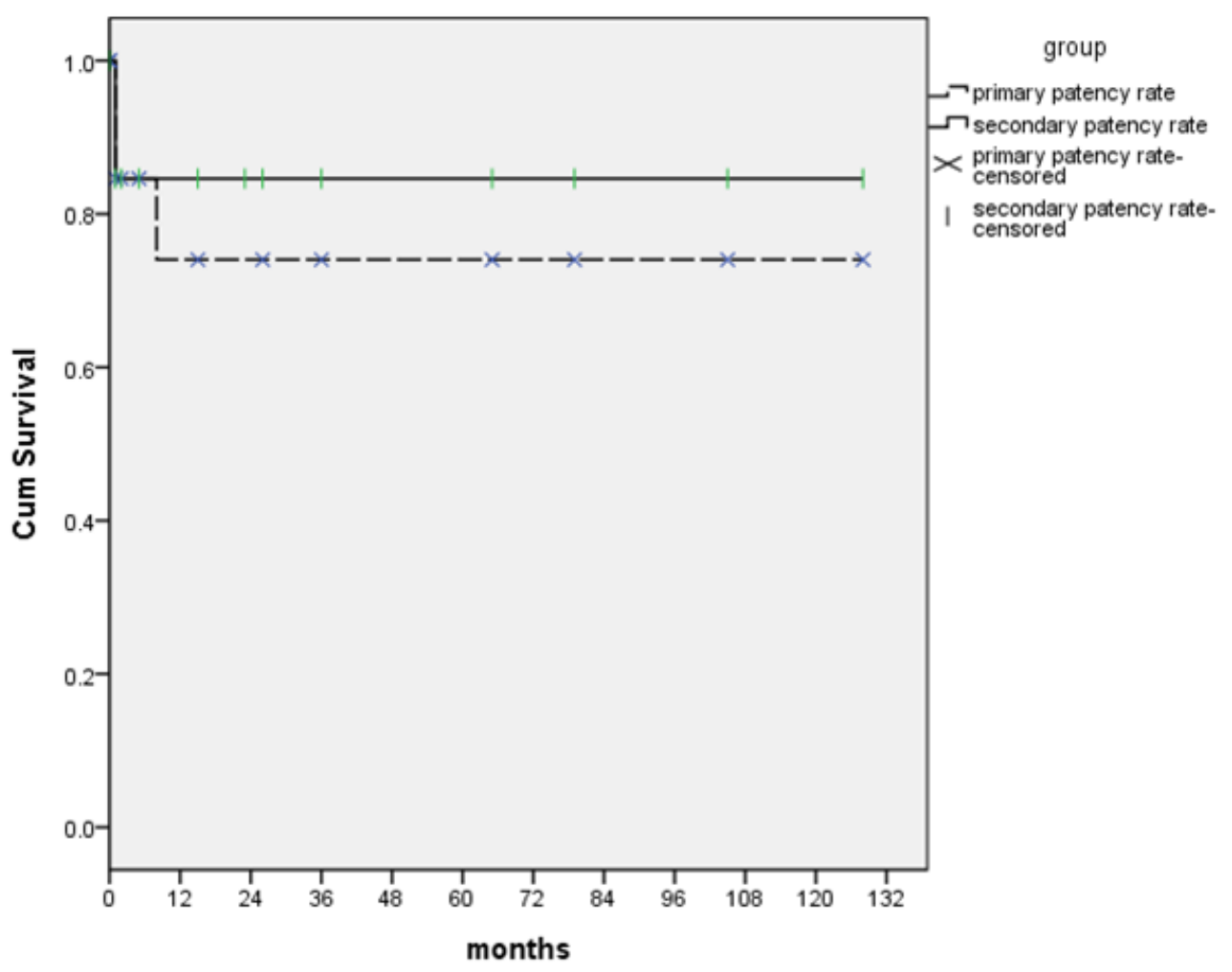

Figure 5 
The primary and secondary patency of the PMVT after local CDT.

Page 14/14 